\title{
Separation of Disaccharides by Comprehensive Two-Dimensional Gas Chromatography-Time-of-Flight Mass Spectrometry. Application to Honey Analysis
}

\author{
Michat Brokl, Ana C. Soria, * Ana I. Ruiz-Matute, María Luz Sanz, and \\ LOURDES RAMOS
}

Instituto de Química Orgánica General (CSIC), Juan de la Cierva 3, 28006 Madrid, Spain

\begin{abstract}
A new method based on comprehensive two-dimensional gas chromatography coupled to time-offlight mass spectrometry ( $\mathrm{GC} \times \mathrm{GC}-\mathrm{ToF} \mathrm{MS}$ ) has been developed for the first time for the analysis of complex mixtures of disaccharides previously converted to their trimethylsilyl oximes (TMSO). Among the different experimental parameters considered for optimization, both the column set combination and the dimensions of the second-dimension column were found to be the most significant with regard to the complete resolution of structurally similar disaccharides. Application of the optimized method to honey analysis allowed the separation of most of the honey disaccharides previously described in the literature. Furthermore, 12 other unknown disaccharides have been separated by this method and characterized from their mass spectral data.
\end{abstract}

KEYWORDS: GC $\times$ GC-ToF MS; disaccharides; trimethylsilyl oximes; honey

\section{INTRODUCTION}

The analysis of low molecular weight carbohydrates is frequently carried out by gas chromatography (GC), although previous conversion of these compounds into volatile derivatives is required. Several derivatization methods have been reported in the literature, including trimethylsilyl ethers (I), trifluoroacetates (2), and alditol acetates (3). Trimethylsilylation (TMS) is the most common procedure because of the simplicity of preparation and easy data interpretation (4); however, reducing sugars give rise to multiple peaks due to the presence of $\alpha$ - and $\beta$-anomers, pyranoside and furanoside rings, and an open chain, which may result in extremely complex chromatographic profiles for carbohydrate mixtures. Oximation of the free carbonyl group of reducing sugars prior to silylation in trimethylsilyl oximes (TMSO) suppresses the anomeric center, and only two derivatives, syn- $(E)$ and anti- $(Z)$, are obtained (5). For nonreducing sugars a single peak corresponding to per-TMS ethers is produced.

However, when complex mixtures of carbohydrates need to be analyzed, this simple procedure is not enough, and chromatographic peak coelutions are observed. Alternative methods, a double-derivatization method aimed to obtain two different chromatographic profiles from the same sample (6) or the use of two columns with different stationary phases $(100 \%$ dimethyl polysiloxane and $35 \%$ dimethyl $-65 \%$ diphenyl polysiloxane) (7), have been proposed to sort out this problem. Although both methods showed some advantages, neither provided a complete resolution of all carbohydrates under analysis.

Comprehensive two-dimensional gas chromatography $(\mathrm{GC} \times \mathrm{GC})$ has recently become a well-established technique for the analysis

*Corresponding author (phone +34 915622900, ext. 306; fax +34 91 5644853; e-mail acsoria@iqog.csic.es). of a diverse range of complex mixtures $(8,9)$. $\mathrm{GC} \times \mathrm{GC}$ adds a second dimension of chromatographic resolution by using two distinct stationary phases (such as polar and nonpolar) within a single analysis. As compared to one-dimensional gas chromatography (1D GC), the use of two separation mechanisms usually results in a significant increase of peak capacity, whereas modulation can provide an improvement in sensitivity, resulting in improved detection of minor compounds. Coupling this technique to time-of-flight mass spectrometry $(\mathrm{GC} \times \mathrm{GC}-\mathrm{ToF} \mathrm{MS})$ offers additional information (mass spectrum) for the identification of unknown components. Therefore, and taking into account all the advantages above exposed it would be interesting to evaluate the potential of $\mathrm{GC} \times \mathrm{GC}-\mathrm{ToF}$ MS in the analysis of complex saccharide mixtures such as honey.

Honey, the natural product made by honeybees from the nectar of flowers, the secretions of living parts of plants, or the excretions of plant-sucking insects, is one of the most complex mixtures of carbohydrates known, mostly consisting of monosaccharides (glucose around 30\% and fructose around 38\%), disaccharides, and oligosaccharides (10). The latter two are composed of glucose and fructose units with glycosidic bonds at different positions and yielding different configurations. A total of 16 disaccharides and 12 trisaccharides have been previously identified and quantified in honey from chromatographic and mass spectrometric data $(7,11,12)$. However, and despite many attempts reported in the literature $(7,13-15)$, complete separation by $1 \mathrm{D}$ GC of the highly complicated isomeric mixture of disaccharides present in honey has not been achieved yet.

Therefore, the general aim of this work has been the development of a new methodology based on GC $\times$ GC-ToF MS for the separation of complex mixtures of disaccharide standards previously converted into TMSO derivatives. To the best of our knowledge, this 
is the first time that a GC $\times$ GC-based methodology has been used for the analysis of carbohydrates. To explore the full potential of GC $\times$ GC-ToF MS, chromatographic data together with mass spectra have been used for the qualitative analysis of a complex disaccharide mixture such as honey.

\section{MATERIALS AND METHODS}

Standards. With the coelutions previously observed in the literature (7) taken into consideration, three mixtures of disaccharide standards (M1-M3) were prepared for $\mathrm{GC} \times \mathrm{GC}$ analysis. In addition, sucrose, $\alpha, \alpha$-trehalose, $\alpha, \beta$-trehalose, inulobiose, and palatinose were individually analyzed. Details concerning standards and the composition of each mixture are summarized in Table $\mathbf{1}$.

Samples. Investigated samples include five artisanal multifloral honeys from the Czech Republic, kindly provided by Dr. Dalibor Titera (Bee Research Institute DOL, Czech Republic), and six commercially available honeys labeled as borage (Borago officinalis), clover (Trifolium sp.),

Table 1. Disaccharide Standards under Analysis

\begin{tabular}{|c|c|c|c|}
\hline mixture & peak & disaccharide & structure and supplier $^{a}$ \\
\hline \multirow[t]{8}{*}{ M1 } & 1 & cellobiose $E$ & 4-O- $\beta$-D-glucopyranosyl-D-glucose (a) \\
\hline & 2 & cellobiose $Z$ & \\
\hline & 3 & maltose $E$ & 4-O- $\alpha$-D-glucopyranosyl-D-glucose (a) \\
\hline & 4 & maltose $Z$ & \\
\hline & 5 & maltulose 1 & 4-O- $\alpha$-D-glucopyranosyl-D-fructose (b) \\
\hline & 6 & maltulose 2 & \\
\hline & 7 & leucrose 1 & 5-O- $\alpha$-D-glucopyranosyl-D-fructose (c) \\
\hline & 8 & leucrose 2 & \\
\hline \multirow[t]{10}{*}{ M2 } & 9 & kojibiose $E$ & 2-O- $\alpha$-D-glucopyranosyl-D-glucose (a) \\
\hline & 10 & kojibiose $Z$ & \\
\hline & 11 & laminaribiose $E$ & 3-O- $\beta$-D-glucopyranosyl-D-glucose (a) \\
\hline & 12 & laminaribiose $Z$ & \\
\hline & 13 & nigerose $E$ & 3-O- $\alpha$-D-glucopyranosyl-D-glucose (a) \\
\hline & 14 & nigerose $Z$ & \\
\hline & 15 & turanose 1 & 3-O- $\alpha$-D-glucopyranosyl-D-fructose (c) \\
\hline & 16 & turanose 2 & \\
\hline & 17 & trehalulose 1 & 1-O- $\alpha$-glucopyranosyl-1-D-fructose (d) \\
\hline & 18 & trehalulose 2 & \\
\hline \multirow[t]{6}{*}{ M3 } & 19 & gentiobiose $E$ & 6-O- $\beta$-D-glucopyranosyl-D-glucose (a) \\
\hline & 20 & gentiobiose $Z$ & \\
\hline & 21 & isomaltose $E$ & 6-O- $\alpha$-D-glucopyranosyl-D-glucose (a) \\
\hline & 22 & isomaltose $Z$ & \\
\hline & 23 & melibiose $E$ & 6-O- $\alpha$-D-galactopyranosyl-D-glucose (c) \\
\hline & 24 & melibiose $Z$ & \\
\hline $1^{b}$ & 25 & sucrose & 2- - - $\alpha-D-g$ lucopyranosyl- $\alpha-D-$-fructofuranoside (a) \\
\hline I & 26 & $\alpha, \alpha$-trehalose & 1-O- $\alpha$-D-glucopyranosyl- $\alpha$-D-glucopyranoside (c) \\
\hline I & 27 & $\alpha, \beta$-trehalose & 1-O- $\alpha$-D-glucopyranosyl- $\beta$-D-glucopyranoside (c) \\
\hline I & 28 & inulobiose & 2-O- $\alpha$-D-fructofuranosyl- $\alpha$-D-fructofuranose (e) \\
\hline I & 29 & palatinose 1 & 6-O- $\alpha-\mathrm{D}$-glucopyranosyl-D-fructose (c) \\
\hline I & 30 & palatinose 2 & \\
\hline
\end{tabular}

a Supplier: (a) Sigma Chemical Co. (St. Louis, MO); (b) Aldrich Chemical Co. (Milwaukee, WI); (c) Fluka (Madrid, Spain); (d) Dr. W. Wach (Südzucker AG, Mannheim, Germany); (e) Beneo P95 Orafti (Tienen, Belgium). ${ }^{b}$ I, individual standard. manuka (Leptospermum scoparium), rewarewa (Knightia excelsa), kamahi (Weinmannia racemosa), and tawari (Ixerba brexioides), acquired in a local market in New Zealand.

Derivatization Procedure. Derivatization (oximation plus trimethylsilylation) of disaccharides was carried out according to Sanz et al. (7). Standard mixtures were separately prepared by diluting $10 \mathrm{mg}$ of different saccharides into $10 \mathrm{~mL}$ of ethanol/water $(70: 30, \mathrm{v} / \mathrm{v})$. One milliliter of each solution was mixed with $0.5 \mathrm{~mL}$ of internal standard (phenyl- $\beta$-D-glucoside, $1 \mathrm{mg} \mathrm{mL}^{-1}$ ). The mixture was evaporated to dryness under vacuum. Oximes were formed by the addition of $350 \mu \mathrm{L}$ of $2.5 \%$ hydroxylamine chloride in pyridine and heating for $30 \mathrm{~min}$ at $75^{\circ} \mathrm{C}$. Silylation of these derivatives was further performed by adding $350 \mu \mathrm{L}$ of hexamethyldisilazane and $35 \mu \mathrm{L}$ of trifluoroacetic acid and heating for $30 \mathrm{~min}$ at $45^{\circ} \mathrm{C}$. After reaction, samples were centrifuged at $8000 \mathrm{rpm}$ for $10 \mathrm{~min}$, and supernatant was taken for analysis. To eliminate the excess of derivatization reagents, supernatant was submitted to a liquid-liquid extraction using hexane/water $(1: 1, \mathrm{v} / \mathrm{v})$. After vortex mixing and separation of phases, the organic phase was used for analysis.

Honey $(0.5 \mathrm{~g})$ was diluted in $25 \mathrm{~mL}$ of an ethanol/water solution $(70: 30, v / v)$, and $1 \mathrm{~mL}$ was evaporated under vacuum. Derivatization conditions were identical to those described above.

Preliminary GC Analysis. GC analyses were carried out on a gas chromatograph equipped with a flame ionization detector (HewlettPackard 5890, Palo Alto, CA), using nitrogen as carrier gas. Column flow was adjusted to the Van Deemter optimum. Three columns of different polarity, ranging from nonpolar dimethyl polysiloxane to polar polyethylene glycol (Table 2), were selected for GC analysis on the basis of previously reported results for carbohydrate separation $(5,7)$ and with the aim of covering a wide range of polarities to achieve the desirable orthogonality between first and second dimensions in subsequent $\mathrm{GC} \times \mathrm{GC}$ analysis. The oven was set at $220{ }^{\circ} \mathrm{C}$ for the BPX50 and Carbowax columns and at $270{ }^{\circ} \mathrm{C}$ for the SPB-1 column (Table 2). Injector (split ratio, 1:20) and detector temperatures were set at $300^{\circ} \mathrm{C}$. Chromatographic peaks were measured using a Chrom-Card 1.20 acquisition system (CE Instruments, Milan, Italy).

GC $\times$ GC Analysis. The GC $\times$ GC-ToF MS system consisted of an HP 6890 gas chromatograph (Agilent Technologies) and a Pegasus 4D ToF MS (LECO Corp., St. Joseph, MI) operating in electron impact (EI) mode at $70 \mathrm{eV}$. The system was equipped with a secondary oven for independent temperature program of the second-dimension column and a quad-jet dual-stage cryomodulator. Liquid nitrogen was used for cryofocusing of the analytes eluting from the first dimension (1D) column, whereas resistively heated air, typically with a temperature offset of $45-60{ }^{\circ} \mathrm{C}$ above that of the main oven, was used for releasing and reinjecting these compounds onto the second dimension (2D) column. In all instances, a minimum of four modulations per peak were obtained.

Table 2 summarizes the stationary phases considered as first and second dimensions in the present study as well as their corresponding dimensions. Helium was used as carrier gas, and all experiments were carried out under a constant pressure regime. Due to the variety of column dimensions assayed, the optimum helium flow rate was calculated for each column combination according to the method of Beens et al. (16). Injection was carried out in the split mode (split ratio, 1:20) at a temperature of $300^{\circ} \mathrm{C}$. The temperatures of the interface and ion source were 250 and $200{ }^{\circ} \mathrm{C}$, respectively. Three column sets (see Table 3 ) were assayed to determine the best combination for disaccharide separation. Chromatographic conditions were optimized for each column set to ensure both optimal carbohydrate separation and column integrity in terms of thermal stability.

Table 2. Columns Used in $\mathrm{GC}$ and $\mathrm{GC} \times \mathrm{GC}$ Analyses

\begin{tabular}{|c|c|c|}
\hline chromatographic technique & stationary phase and supplier ${ }^{a}$ & column dimensions $\left(\right.$ length $(\mathrm{m}) \times$ i.d. $\left.(\mathrm{mm}) \times d_{f}(\mu \mathrm{m})\right)$ \\
\hline \multirow[t]{3}{*}{$\mathrm{GC}$ or $1 \mathrm{D} \mathrm{GC} \times \mathrm{GC}$} & SPB-1 (dimethyl polysiloxane) (a) & $30 \times 0.25 \times 0.1$ \\
\hline & BPX50 (50\% phenyl polysilphenylene-siloxane) (b) & $30 \times 0.25 \times 0.25$ \\
\hline & Carbowax (polyethylene glycol) (c) & $10 \times 0.25 \times 0.125$ \\
\hline \multirow[t]{3}{*}{$2 \mathrm{D} G \mathrm{GC} \times \mathrm{GC}$} & EQUITY-1 (dimethyl polysiloxane) (a) & $1.2 \times 0.1 \times 0.1$ \\
\hline & SPB-1 (dimethyl polysiloxane) (a) & $2.2 \times 0.25 \times 0.25$ \\
\hline & BPX50 (50\% phenyl polysilphenylene-siloxane) (b) & $1.2 \times 0.1 \times 0.1$ \\
\hline
\end{tabular}

\footnotetext{
a Supplier: (a) Supelco (Bellefonte, PA); (b) SGE (Europa Ltd., UK); (c) Alltech (Bannockburn, IL).
} 
Table 4. Peak Width (w), Symmetry, and Kovats Retention Index (I) of Selected Disaccharides Used as Preliminary Data for Selection of 1D Column in $\mathrm{GC} \times \mathrm{GC}$ Analysis

\begin{tabular}{lllcccc}
\hline & & & kojibiose & kojibiose \\
$Z$ & parameter & sucrose & $E$ & $\begin{array}{c}\text { isomaltose } \\
Z\end{array}$ & $\begin{array}{c}\text { isomaltose } \\
Z\end{array}$ \\
\hline SPB-1 & $w$ (min) & 0.14 & 0.27 & 0.30 & 0.30 & 0.32 \\
& symmetry & 0.95 & 0.90 & 1.00 & 0.93 & 1.00 \\
& $I$ & 2729 & 2977 & 3024 & 3070 & 3116 \\
BPX50 & $w$ (min) & 0.19 & 0.27 & 0.29 & 0.34 & 0.35 \\
& symmetry & 1.67 & 1.27 & 0.99 & 1.23 & 1.09 \\
& $I$ & 2441 & 2619 & 2652 & 2751 & 2775 \\
& & & & & & \\
Carbowax & $w$ (min) & 0.18 & 0.27 & 0.27 & 0.70 & 0.51 \\
& symmetry & 2.17 & 1.98 & 1.96 & 2.09 & 1.63 \\
& $I$ & 2544 & 2806 & 2830 & 2957 & 2979 \\
\hline
\end{tabular}

Mass spectra were collected in the $m / z$ 50-700 range at an acquisition rate of $50 \mathrm{~Hz}$. Data were recorded and processed using LECO Chroma TOF 3.2 software. Analyte identification was considered to be positive when both retention times and MS data were consistent with those of standards analyzed under identical experimental conditions. Identities were considered to be tentative when only experimental MS data were available and no further comparison was possible due to the lack of standards.

\section{RESULTS AND DISCUSSION}

Development of a $\mathrm{GC} \times \mathrm{GC}$ Method for Disaccharide Analysis. Preliminary experiments in 1D GC were carried out to determine the chromatographic behavior of TMSO disaccharides in the three phases considered as first dimension in $\mathrm{GC} \times \mathrm{GC}$ analyses (Table 2). Parameters calculated for each disaccharide at the temperatures indicated under Materials and Methods included Kovats retention index $(I)$, peak width $(w)$, and symmetry, as measured by the tailing factor from peak width values at $5 \%$ peak height.

As an example, Table 4 shows these values for sucrose, as the first disaccharide to elute, kojibiose, which generally elutes at a mid-retention time, and isomaltose, as the last eluting carbohydrate in all three assayed columns. In general, peak widths of TMSO disaccharides were adequate for the three columns tested, their values ranging from 0.14 to $0.35 \mathrm{~min}$, with the exception of those values found for the most retained carbohydrates in Carbowax, which showed $w$ values of $0.50-0.70$ min. Both SPB-1 and BPX50 columns provided very symmetric peaks, whereas strong tailing was observed for those peaks eluted in Carbowax column. Moreover, the negative effect of silylation reagents on the polyethylene glycol phase made this column inappropriate for TMSO disaccharide separation.

Therefore, two column sets were considered for further $\mathrm{GC} \times \mathrm{GC}$ optimization: SPB-1 $\times$ BPX50 (nonpolar $\times$ moderately polar) and BPX50 $\times$ EQUITY-1 (moderately polar $\times$ nonpolar); details on stationary phases and dimensions of $1 \mathrm{D}$ and 2D columns are listed in Table 2. Mixtures of TMSO disaccharides M1, M2, and M3 were used to optimize the separation. Different experimental conditions such as modulation time $(3-7 \mathrm{~s})$, hot jet time $(0.6-1.4 \mathrm{~s})$, and temperature offset $\left(30-60{ }^{\circ} \mathrm{C}\right.$ over the main oven) were evaluated. Moreover, different temperature ramps in $1 \mathrm{D}$ and 2D were assayed to select the optimum for each column combination. The finally proposed experimental conditions are summarized in Table 3.

Figure 1A shows the GC $\times$ GC-ToF MS contour plot of $m / z 361$ (characteristic of silylated saccharides) for mixtures M1, M2, and M3 using the SPB-1 $\times$ BPX50 column set. In general, the coupling of both columns did not provide a noticeably good separation of disaccharides, and no advantages were observed with this 


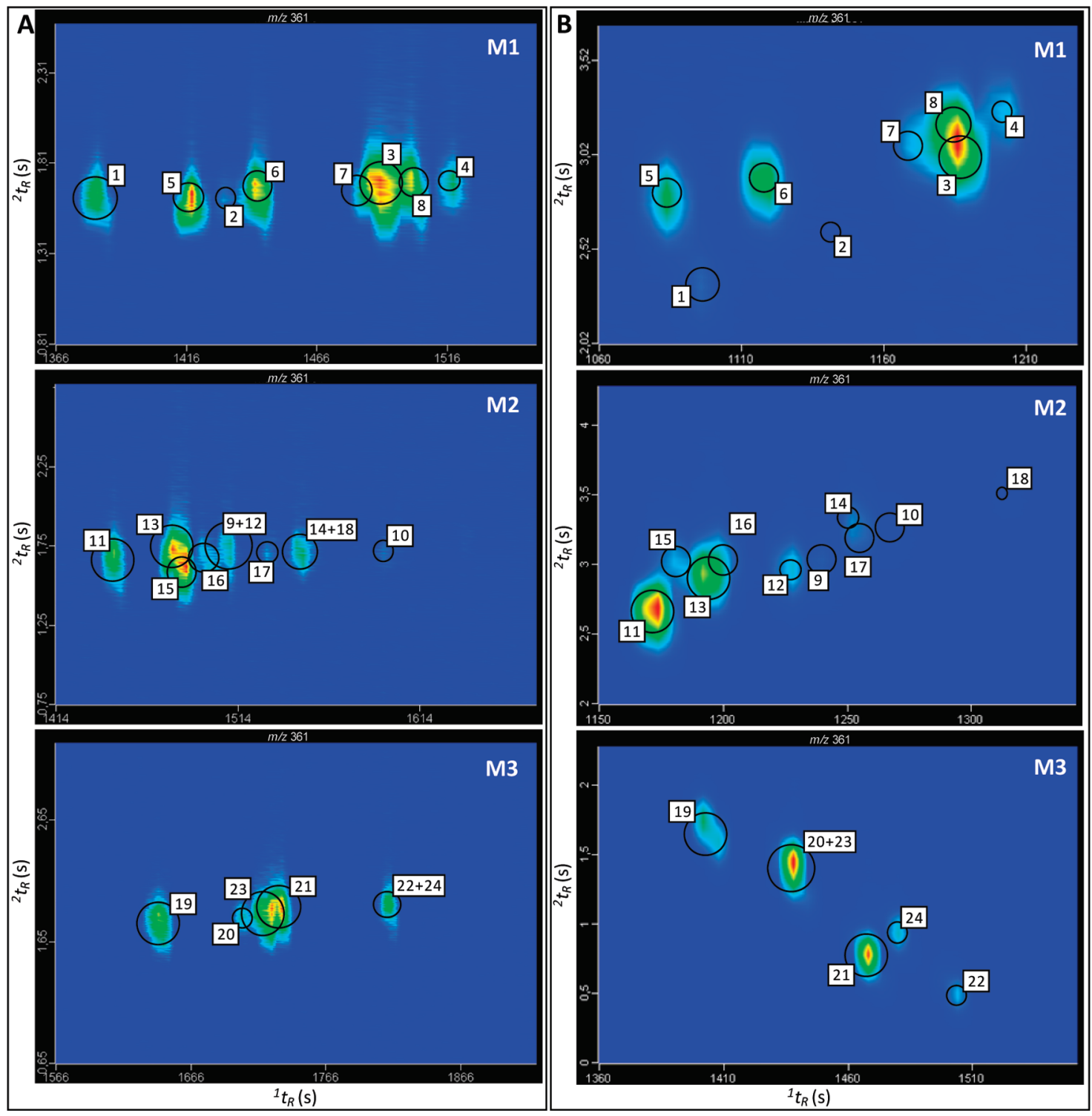

Figure 1. $\mathrm{GC} \times \mathrm{GC}$ contour plots $(\mathrm{m} / \mathrm{z} 361)$ of standard disaccharide mixtures $(\mathrm{M} 1, \mathrm{M} 2$, and $\mathrm{M} 3)$ on column sets $(\mathbf{A}) \mathrm{SPB}-1 \times \mathrm{BPX} 50$ and $(\mathbf{B}) \mathrm{BPX} 50 \times$ EQUITY-1. Peak and mixture numbers refer to those of Table 1.

column combination over that achieved in the 1D GC. Only a slight separation of peaks 13 and 15 (nigerose $E$ and turanose 1) was obtained. Therefore, this column set proved not to be appropriate for disaccharide separation.

Although a polar $\times$ nonpolar column set is not the most common approach for $\mathrm{GC} \times \mathrm{GC}$ analysis, this combination has been reported to be useful for the separation of different compounds such as amino acids or volatiles of roasted coffee. As can be observed in Figure 1B, the resolution of test disaccharides with the BPX50 $\times$ EQUITY-1 column set was superior to that achieved with the previous combination. Although overlapping of some compounds such as maltose $E$ and leucrose 2 (peaks 3 and 8 ) or gentiobiose $Z$ and melibiose $E$ (peaks 20 and 23) was still observed after optimization of experimental conditions, this column combination resulted in a general improvement of distribution of peaks across the 2D plane irrespective of the mixture considered. Therefore, this set was chosen for further analyses.

Because of the usual differences in concentration levels of target compounds and the interest in proper detection of minor (and/or new) disaccharides that could be present in real-life samples, the possibility of increasing the loading capacity of the $2 \mathrm{D}$ column was evaluated. On the basis of data previously reported in the literature $(17,18)$, the use of a longer and wider bore $2 \mathrm{D}$ column was expected to be a valuable analytical alternative to achieve this goal. Thereby, in this new set of experiments, the SPB-1 column with $2.2 \mathrm{~m} \times 0.25 \mathrm{~mm}$ i.d. $\times 0.25 \mu \mathrm{m} d_{f}$ dimensions was used for $2 \mathrm{D}$, and results were compared with those obtained with the BPX50 $\times$ EQUITY-1 column set $(2 \mathrm{D}$ column: $1.2 \mathrm{~m} \times 0.1 \mathrm{~mm}$ i.d. $\times 0.1 \mu \mathrm{m} d_{f}$ ).

As expected (17), the vacuum outlet pressure in the $\mathrm{GC} \times \mathrm{GC}$ ToF MS instrument at operating conditions near the optimum linear velocities in the $1 \mathrm{D}$ and $2 \mathrm{D}$ resulted in a lower average column pressure in the $2 \mathrm{D}$. Thus, acceptable values for the analyte retention times in both dimensions in BPX50 $\times$ SPB-1, as compared to those of BPX $50 \times$ EQUITY-1, were obtained by selecting the appropriate flow rate according to ref 16 . The higher loading capacity of the former column combination resulted indeed in an improved detection of some less abundant (well separated) components in the test mixtures. Unfortunately, it also led to broad chromatographic peaks in the case of analytes at higher concentrations, resulting in a relatively higher number of 


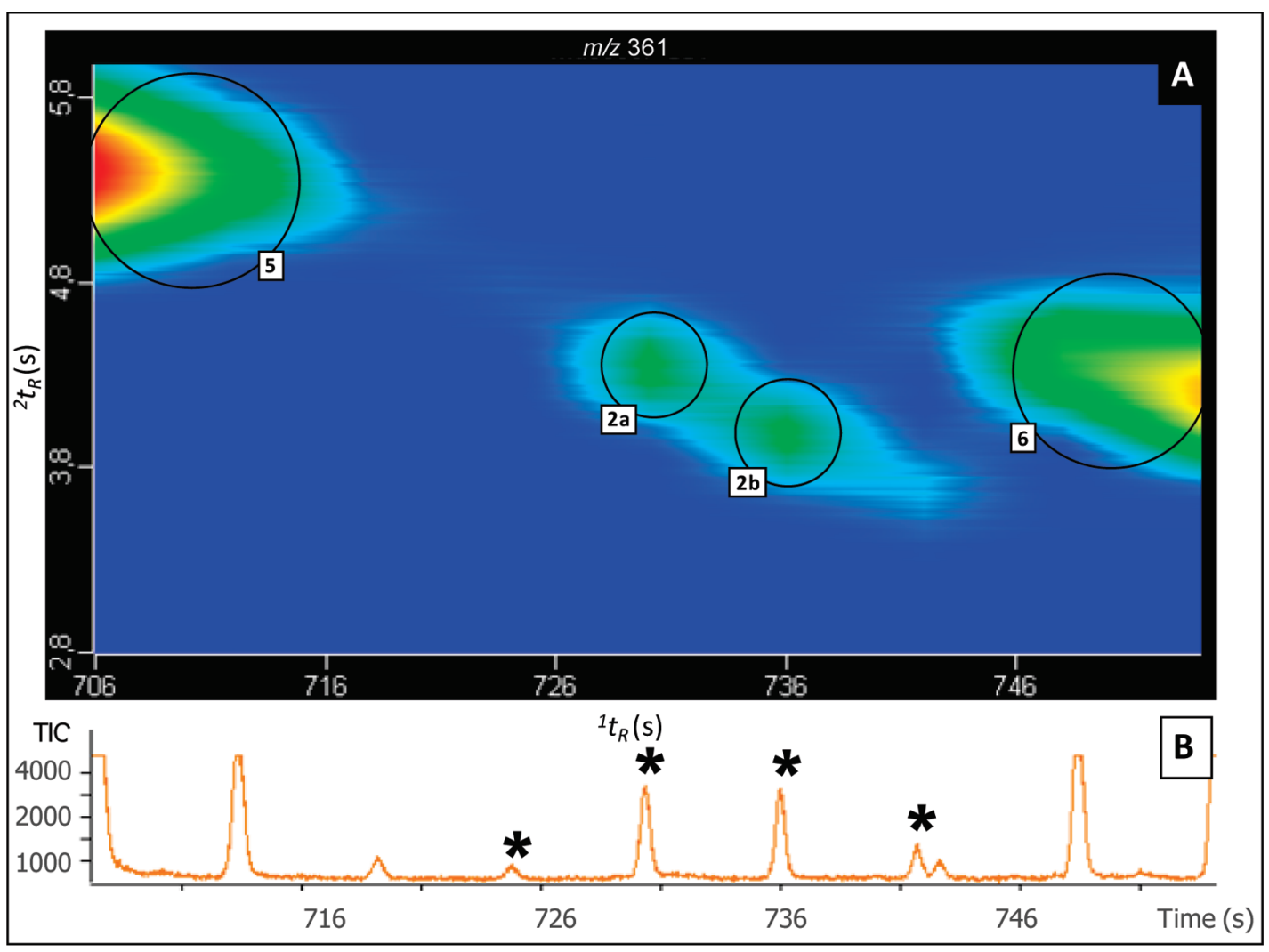

Figure 2. (A) $\mathrm{GC} \times \mathrm{GC}$ contour plot ( $\mathrm{m} / \mathrm{z} 361$ ) of cellobiose $Z$ (peaks $2 \mathrm{a}$ and $2 \mathrm{~b}$ ) standard (component of mixture M1) analyzed on BPX50 $\times$ SPB-1 and (B) corresponding raw data obtained for this single compound (asterisks show the position of the modulations demonstrating that only one peak was actually present). Peak numbers refer to those of Table 1.

modulations per peak. This effect, combined with the relatively fast temperature ramps applied in this study to ensure rapid elution of the analytes, resulted in consecutive modulated fractions of a peak eluting at increasingly earlier elution times from the second dimension. In an extreme case, such as that shown in Figure 2A for cellobiose $Z$, two apparent maxima (i.e., peaks $2 \mathrm{a}$ and $2 \mathrm{~b}$ ) can be differentiated in the contour plot, whereas careful inspection of the raw data (Figure 2B) proves that these two maxima in fact correspond to a single compound. The analysis of the corresponding individual standard under these conditions resulted in a similar visual effect in the contour plot. As the use of a slower temperature ramp did not solve the problem either, the BPX50 $\times$ EQUITY-1 combination with a narrow 2D column was selected for further application to honey analysis.

Analysis of Honey Disaccharides. To evaluate the potential advantages of the developed $\mathrm{GC} \times \mathrm{GC}$ method for disaccharide separation, 11 honey samples were analyzed using column set BPX50 $\times$ EQUITY-1. Similar qualitative profiles were obtained for all of the analyzed samples, and only variations in the relative abundances of disaccharides were observed. As an example, Figure 3 shows the $\mathrm{GC} \times \mathrm{GC}$ contour plot of the manuka honey under analysis.

Except for peaks 3, 4, 13, 15, and 16, corresponding to maltose $E$ and $Z$, nigerose $E$, and turanose 1 and 2, which remained unresolved, the rest of the previously identified disaccharides of honey were chromatographically resolved; therefore, this $\mathrm{GC} \times \mathrm{GC}$ method clearly improved disaccharide resolution over previous 1D GC methodologies (7). In addition, the extraction of characteristic MS ion fragments (Figure 3C) allowed the individual detection of turanose $(\mathrm{m} / \mathrm{z} 307)$ from maltose and nigerose $(\mathrm{m} / \mathrm{z}$ 319) and even the clear separation of both isomers of turanose (peaks 15 and 16) and maltose (peaks 3 and 4). Only maltose
$E$ and nigerose $E$ (peaks 3 and 13) were considered to coelute, due to their similar mass spectra and close retention times.

Moreover, the optimized GC $\times$ GC-ToF MS method allowed the separation of several unknown compounds (peaks labeled $U$ in Figure 3), which have not been previously identified in honey, probably due to overlap with other disaccharides in 1D GC chromatograms. Table $\mathbf{5}$ shows the relative abundances of the characteristic $m / z$ ions of the unknown carbohydrates detected in the 11 honeys investigated. Although all of these compounds showed a mass spectrum typical of disaccharides, their characterization based on mass spectral data was only tentative because no commercial standards were available to confirm their identities.

With the exception of U12, the unknown disaccharides eluted at shorter retention times in $1 \mathrm{D}(16-18 \mathrm{~min})$ than identified disaccharides (excluding sucrose). Previous works have indicated that nonreducing sugars or disaccharides with two fructose groups, such as inulobiose, elute in this area $(7,11)$.

Peaks U1, U2, and U3 showed relatively high fragment ions at $m / z 437$, which is characteristic of ketohexoses in both pyranose and furanose forms, free and monosubstituted (19), and at $m / z$ 307 , comparable to 361 , which is indicative of a ketose oxime, probably fructose, linked to the nonreducing unit through the hydroxyl group in position 1 or 3 . Therefore, these compounds could tentatively be assigned as $(2 \rightarrow 1)$ and/or $(2 \rightarrow 3)$ fructosylfructoses.

Peaks U4 and U5 had a very abundant fragment at $m / z$ 217, which could proceed from the oxime chain or from a fructose ring. A fragment at $m / z 538$ in U5 confirmed the presence of an oxime. These compounds may correspond to reducing disaccharides.

Peaks U6-U9, U11, and U12 showed mass spectra with a characteristic distribution of ions at $m / z$ 191, 204, and 217; U10 showed a relatively high fragment ion at $m / z 437$ and characteristic 

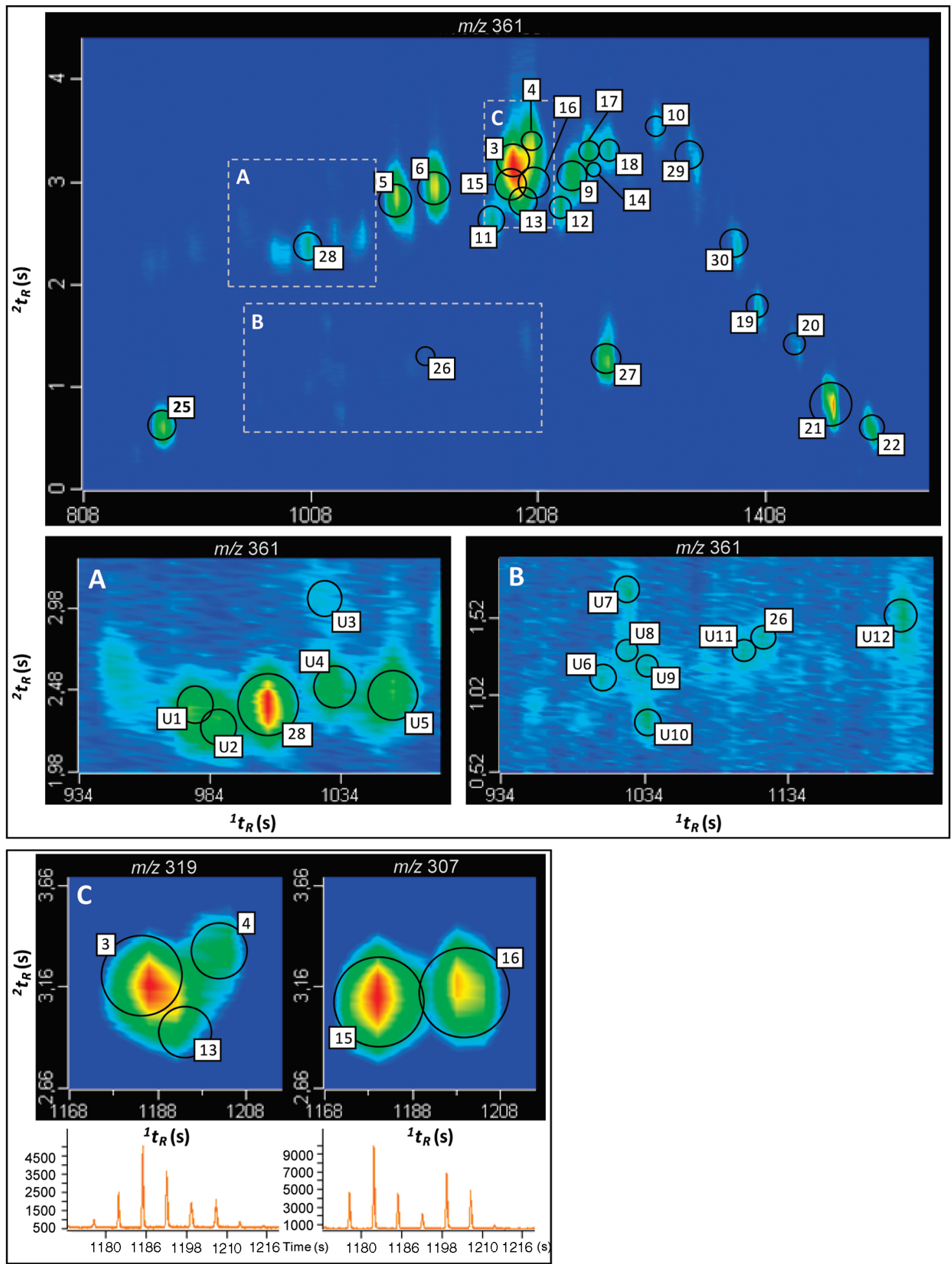

Figure 3. $\mathrm{GC} \times \mathrm{GC}$ contour plot $(\mathrm{m} / \mathrm{z} 361)$ of a manuka honey sample analyzed on column set $\mathrm{BPX} 50 \times \mathrm{EQUITY}-1$. Insets $A$ and $B$ correspond to $\mathrm{m} / z 361$; inset $C$ corresponds to contour plots $\mathrm{m} / \mathrm{z} 319$ and 307 . Corresponding raw data are shown below these figures. Peak numbers refer to those of Table 1 . U1-U12 are unknown disaccharides.

fragments at $m / z 243$ and 271 . Further studies are required to confirm these structures.

A GC $\times$ GC-ToF MS-based method has been developed for the analysis of complex mixtures of disaccharides. Among the different operating conditions under optimization, careful selection of the column set and of the dimensions of the 2D has been shown to be crucial for an improved separation of carbohydrates and for obtaining single and unequivocal signals for every analyte. The performance of the optimized method has been demonstrated by successful application to the challenging separation of honey disaccharides. As far as we know, this is the first time that $\mathrm{GC} \times \mathrm{GC}-\mathrm{ToF}$ MS has been applied to carbohydrate analysis. The improved resolution over 1D GC and the possibility of characterization of unknown carbohydrates based on mass spectral data make the developed methodology a valuable tool to be considered in disaccharide analysis of other complex matrices. 
Table 5. Relative Abundance of Characteristic $m / z$ Ratios of Unknown Disaccharides U1-U12 in Honey ${ }^{a}$

\begin{tabular}{|c|c|c|c|c|c|c|c|c|c|c|c|c|c|c|c|c|}
\hline \multirow[b]{2}{*}{ peak } & \multicolumn{16}{|c|}{$\mathrm{m} / \mathrm{z}$} \\
\hline & 103 & 147 & 191 & 204 & 205 & 217 & 243 & 271 & 305 & 307 & 319 & 361 & 422 & 437 & 451 & 538 \\
\hline U1 & 100 & 69 & 12 & 1 & 8 & 64 & 0 & 0 & 0 & 6 & 2 & 6 & 0 & 2 & 0 & 1 \\
\hline U2 & 100 & 73 & 7 & 2 & 8 & 68 & 0 & 0 & 0 & 8 & 2 & 8 & 0 & 2 & 1 & 1 \\
\hline U3 & 100 & 79 & 15 & 18 & 12 & 38 & 4 & 0 & 0 & 9 & 3 & 9 & 0 & 3 & 0 & 3 \\
\hline U4 & 100 & 83 & 26 & 4 & 5 & 81 & 3 & 2 & 0 & 0 & 0 & 16 & 0 & 0 & 1 & 0 \\
\hline U5 & 85 & 90 & 13 & 12 & 9 & 100 & 5 & 4 & 0 & 0 & 2 & 20 & 0 & 0 & 0 & 3 \\
\hline U6 & 75 & 100 & 24 & 36 & 12 & 33 & 7 & 7 & 4 & 0 & 0 & 23 & 0 & 0 & 0 & 3 \\
\hline U7 & 89 & 100 & 24 & 13 & 13 & 45 & 10 & 8 & 6 & 0 & 0 & 45 & 0 & 0 & 0 & 0 \\
\hline U8 & 90 & 100 & 15 & 16 & 6 & 32 & 12 & 3 & 0 & 0 & 0 & 18 & 0 & 0 & 0 & 0 \\
\hline U9 & 92 & 100 & 14 & 32 & 11 & 42 & 8 & 15 & 0 & 0 & 7 & 28 & 0 & 0 & 0 & 0 \\
\hline U10 & 78 & 100 & 16 & 18 & 11 & 38 & 16 & 10 & 0 & 0 & 0 & 47 & 0 & 8 & 0 & 0 \\
\hline U11 & 66 & 100 & 48 & 39 & 9 & 54 & 22 & 21 & 0 & 0 & 0 & 29 & 7 & 0 & 0 & 0 \\
\hline U12 & 80 & 100 & 16 & 27 & 29 & 43 & 8 & 4 & 0 & 0 & 0 & 25 & 0 & 0 & 0 & 1 \\
\hline
\end{tabular}

${ }^{a}$ Peaks are labeled as in Figure 3.

\section{ABBREVIATIONS USED}

1D, first dimension; 2D, second dimension; 1D GC, onedimensional gas chromatography; GC $\times$ GC-ToF MS, comprehensive two-dimensional gas chromatography-time-of-flight mass spectrometry; TMSO, trimethylsilyl oximes.

\section{ACKNOWLEDGMENT}

We are grateful to Dr. I. Martínez-Castro and Dr. J. Sanz for critical reading of the manuscript.

\section{NOTE ADDED AFTER ASAP PUBLICATION}

Table 1 was modified, and changes to the reference list were made in the version of this paper published October 25, 2010. The correct version published on October 28, 2010.

\section{LITERATURE CITED}

(1) Sweeley, C. C.; Bentley, R.; Makita, M.; Wells, W. W. Gas-liquid chromatography of trimethylsilyl derivatives of sugars and related substances. J. Am. Chem. Soc. 1963, 85, 2497-2507.

(2) Sullivan, J. E.; Schewe, L. R. Preparation and gas chromatography of highly volatile trifluoroacetylated carbohydrates using N-methyl bis (trifluoroacetamide). J. Chromatogr. Sci. 1977, 15, 196-197.

(3) Blakeney, A. B.; Harris, P. J.; Henry, R. J.; Stone, B. A. A simple and rapid preparation of alditol acetates for monosaccharide analysis. Carbohydr. Res. 1983, 113, 291-299.

(4) Troyano, E.; Olano, A.; Fernández-Díaz, M.; Sanz, J.; Martínez-Castro, I. Gas chromatographic analysis of free monosaccharides in milk. Chromatographia 1991, 32, 379-382.

(5) Molnár-Perl, I.; Horváth, K. Simultaneous quantitation of mono-, di- and trisaccharides as their TMS ether oxime derivatives by GC-MS: I. In model solutions. Chromatographia 1997, 45, 321327.

(6) Mateo, R.; Bosch, F.; Pastor, A. Capillary column gas chromatographic identification of sugars in honey as trimethylsilyl derivatives. J. Chromatogr. A 1987, 410, 319-328.

(7) Sanz, M. L.; Sanz, J.; Martínez-Castro, I. Gas chromatographic-mass spectrometric method for the qualitative and quantitative determination of disaccharides and trisaccharides in honey. J. Chromatogr. A 2004, $1059,143-148$.

(8) Adahchour, M.; Beens, J.; Vreuls, R. J. J.; Brinkman, U. A. Th. Recent developments in comprehensive two-dimensional gas chromatography $(\mathrm{GC} \times \mathrm{GC})$ : I. Introduction and instrumental set-up. Trends Anal. Chem. 2006, 25, 438-454.

(9) Górecki, T.; Harynuk, J.; Panić, O. J. The evolution of comprehensive two-dimensional gas chromatography $(\mathrm{GC} \times \mathrm{GC})$. J. Sep. Sci. 2004, 27, 359-379.
(10) Belitz, H. D.; Groch, W. In Food Chemistry, 2nd ed.; Springer-Verlag: Heidelberg, Germany, 1992.

(11) Ruiz-Matute, A. I.; Sanz, M. L.; Martínez-Castro, I. Use of gas chromatography-mass spectrometry for identification of a new disaccharide in honey. J. Chromatogr. A 2007, 1157, $480-483$.

(12) Brokl, M.; Soria, A. C.; Martínez-Castro, I.; Sanz, M. L.; Ruiz-Matute, A. I. Characterization of $O$-trimethylsilyl oximes of trisaccharides by gas chromatography-mass spectrometry. J. Chromatogr. A 2009, 1216, 4689-4692.

(13) Horváth, K.; Molnár-Perl, I. Simultaneous quantitation of mono-, di-and trisaccharides by GC-MS of their TMS ether oxime derivatives: II. In honey. Chromatographia 1997, 45, 328-335.

(14) Low, N. H.; Sporns, P. Analysis and quantitation of minor di- and trisaccharides in honey, using capillary gas chromatography. J. Food Sci. 1988, 53, 558-561.

(15) de la Fuente, E.; Sanz, M. L.; Martínez-Castro, I.; Sanz, J. Development of a robust method for the quantitative determination of disaccharides in honey by gas chromatography. J. Chromatogr. A 2006, 1135, 212-218.

(16) Beens, J.; Janssen, H. G.; Adahchour, M.; Brinkman, U. A. Th. Flow regime at ambient outlet pressure and its influence in comprehensive two-dimensional gas chromatography. J. Chromatogr. A 2005, 1086, $141-150$.

(17) Mayadunne, R.; Nguyen, T. T.; Marriott, P. J. Amino acid analysis by using comprehensive two-dimensional gas chromatography. Anal. Bioanal. Chem. 2005, 382, 836-847.

(18) Ryan, D.; Shellie, R.; Tranchida, P.; Casilli, A.; Mondello, L.; Marriott, P. Analysis of roasted coffee bean volatiles by using comprehensive two-dimensional gas chromatography-time-offlight mass spectrometry. J. Chromatogr. A 2004, 1054, 57-65.

(19) Koek, M. M.; Muilwijk, B.; van Stee, L. L. P.; Hankemeier, T. Higher mass loadability in comprehensive two-dimensional gas chromatography-mass spectrometry for improved analytical performance in metabolomics analysis. J. Chromatogr. A 2008, 1186, $420-429$.

(20) Hail, M. E.; Yost, R. A. Theoretical and practical aspects of short open tubular columns at subambient pressures in gas chromatography/mass spectrometry. Anal. Chem. 1989, 61, 2402-2410.

(21) Kamerling, J. P.; Vliegenthart, J. F. G.; Vink, J.; de Ridder, J. J. Mass spectrometry of pertrimethylsilyl oligosaccharides containing fructose units. Tetrahedron 1972, 28, 4375-4387.

Received for review July 8, 2010. Revised manuscript received September 29, 2010. Accepted October 5, 2010. This work has been funded by projects CTQ2006-14993/BQU (financed by CICYT) and ANALISYC2 (financed by Comunidad de Madrid). M.B. thanks the Consejería de Educación de la Comunidad de Madrid for a predoctoral contract. 